\title{
Asymptomatic Reactivation of SARS-CoV-2 in a Child with Neuroblastoma Characterised by Whole Genome Sequencing
}

\author{
Satya Yadav ${ }^{1}$, Dhwanee Thakkar ${ }^{2}$, Rahul Bhoyar ${ }^{3}$, Abhinav Jain ${ }^{3}$, Teena Wadhwa ${ }^{1}$, \\ Mohamed Imran ${ }^{3}$, Bani Jolly ${ }^{3}$, Mohit Divakar ${ }^{3}$, Rohit Kapoor ${ }^{1}$, Neha Rastogi ${ }^{1}$, Disha \\ Sharma $^{3}$, Paras Sehgal ${ }^{3}$, Gyan Ranjan ${ }^{3}$, Sridhar Sivasubbu ${ }^{3}$, Smita Sarma ${ }^{1}$, and Vinod \\ Scaria $^{3}$ \\ ${ }^{1}$ Medanta The Medicity \\ ${ }^{2}$ Fortis Memorial Research Institute \\ ${ }^{3}$ CSIR Institute of Genomics \& Integrative Biology
}

October 12,2020

\section{Asymptomatic Reactivation of SARS-CoV-2 in a Child with Neuroblastoma Characterised by Whole Genome Sequencing}

Satya Prakash Yadav ${ }^{1}$, Dhwanee Thakkar ${ }^{1}$, Rahul C. Bhoyar ${ }^{2}$, Abhinav Jain ${ }^{2,3}$, Teena Wadhwa ${ }^{4}$, Mohamed Imran $^{2,3}$, Bani Jolly ${ }^{2,3}$, Mohit Kumar Divakar ${ }^{2,3}$, Rohit Kapoor ${ }^{1}$, Neha Rastogi ${ }^{1}$, Disha Sharma ${ }^{2,3}$, Paras Sehgal $^{2,3}$, Gyan Ranjan ${ }^{2,3}$, Sridhar Sivasubbu ${ }^{2,3}$, Smita Sarma ${ }^{4}$, Vinod Scaria ${ }^{2,3}$

1. Pediatric Hematology Oncology and Bone Marrow Transplant Unit, Cancer Institute, Medanta The Medicity, Gurgaon, Haryana, India.

2. CSIR Institute of Genomics and Integrative Biology (CSIR-IGIB), Mathura Road, Delhi, India

3. Academy of Scientific and Innovative Research (AcSIR), Ghaziabad- 201002, India

4. Department of Microbiology, Medanta The Medicity, Gurgaon, Haryana, India.

Correspondence : Dr Satya P Yadav, Pediatric Hematology Oncology \& BMT Unit, Cancer Institute, Medanta -The Medicity, Gurgaon, Haryana, India 122001.

Email:satya_1026@hotmail.com, Fax: +91 1244962222

Total Pages - 4

Abstract (word count) - N/A

Table - 0

Figure - 1

Text (word count) -479

Running title-COVID-19 reactivation proven by sequencing

Key words - COVID-19; Reactivation; SARS-CoV-2; Genome Sequencing; Neuroblastoma

To the editor:

Corona virus disease 2019 (COVID-19) in children with cancer is usually mild and most children recover without any treatment $(1,2)$. To et al. and others (3-6) have recently reported reinfections with severe acute respiratory syndrome corona virus -2 (SARS-CoV-2) confirmed by genome sequencing. A few recent cases of 
potential reactivation/reinfection with SARS-CoV-2 have been reported in cancer patients on chemotherapy (7-9), however were not confirmed by genetic characterization of the virus. Here we report results of genome sequencing in child with neuroblastoma reported previously by us as re-infected with SARS-CoV-2 after recovering fully and developing IgG antibodies (8). Genetic characterization of the virus from both episodes confirmed reactivation.

The 3-year-old boy with stage 4 neuroblastoma was being treated as per OPEC/OJEC protocol. After 4 cycles of chemotherapy and surgery he was found to be positive on real time-PCR(RT-PCR) assay for SARS-CoV-2 on on nasopharyngeal \& throat swabs (Thermofisher TaqPath; Ct values N-14.59 ORF-16.84 S-17.48). As he was asymptomatic, was isolated at home and the chemotherapy was postponed. Three weeks later, the repeat RT-PCR assay was negative and was commenced on the next course of chemotherapy OJEC. Six weeks later, prior to course of next chemotherapy, the child tested positive again on routine testing for SARS-CoV-2 RT-PCR assay (Siemens Labgun ExoFast; Ct values, N-22.37 RdRp-22.15). The quantitative IgG antibody assay (by CLIA for anti S1 and anti S2 specific IgG; $306 \mathrm{AU} / \mathrm{ml}$ ) was also positive. The gap between first and second episodes was 42 days. He was isolated at home for another 2 weeks, and chemotherapy was resumed after testing negative on RT-PCR (8).

The archived RNA samples were used for the library preparation using the COVID Seq assay kit which utilizes the set of 98 primers specific for the SARS-CoV-2 genome (10). The synthesized libraries were sequenced on the MiSeq sequencer (Illumina, Inc) on a $75 \times 2$ paired end sequencing recipe. Genomes were assembled using a reference-based approach following a previously published protocol (11). Briefly, quality control of the FASTQ files was performed followed by adapter/base trimming at a Phred quality score of Q30 and length cut-off 70 bps. Reads were aligned to the SARS-CoV-2 reference genome (NC_045512.2) The genomes were assembled at a depth of $12615.92 \mathrm{X}$ and $7834.78 \mathrm{X}$, with a genome coverage of $99.84 \%$ and $99.70 \%$ for the 2 episodes E1 and E2 respectively (Fig.1). Analysis revealed 14 variants in the genome isolate from E1. 13 out of the 14 variants from E1 were also present in the isolate from $\mathrm{E} 2$ while 1 variant $12793 \mathrm{G}>\mathrm{A}$ was present in E2 with a read count proportion of 35.8\%. Of the 14 variants, 6 were non-synonymous.

Put together, the genomic analysis suggests that the virus isolated from the two episodes were not distinctly different, suggesting reactivation of the virus during the course of chemotherapy. To the best of our knowledge, this is one of the earliest cases of genetically characterized reactivation of SARS-CoV-2.

Disclosure - All authors have nothing to declare. All authors have contributed to the manuscript.

\section{References}

1. Yadav SP. COVID-19 in Children with Blood and Cancer Disorders: What Do We Know So Far? $J$ Pediatr Hematol Oncol . 2020;42(6):413-414. doi:10.1097/MPH.0000000000001872

2. Boulad F, Kamboj M, Bouvier N, Maureen A, Kung AL. COVID-19 in Children with Cancer in New York City. JAMA Oncology . doi:10.1001/jamaoncol.2020.2028.

3. To KK, Hung IF, Ip JD, Chu AW, Chan W, Tam AR et al. COVID-19 re-infection by a phylogenetically distinct SARS-coronavirus-2 strain confirmed by whole genome sequencing. Clinical Infectious Diseases , ciaa1275, https://doi.org/10.1093/cid/ciaa1275

4. Van Elslande J, Vermeersch P, Vandervoort K, Wawina-Bokalanga T, Vanmechelen B, Wollants E, Laenen L, André E, Van Ranst M, Lagrou K, Maes P. Symptomatic SARS-CoV-2 reinfection by a phylogenetically distinct strain. Clin Infect Dis. 2020 Sep 5:ciaa1330. doi: 10.1093/cid/ciaa1330. Epub ahead of print. PMID: 32887979; PMCID: PMC7499557.

5. Tillett R, Sevinsky J, Hartley P, et al. Genomic Evidence for a Case of Reinfection with SARS-CoV-2. 2020 ; Available at: https://papers.ssrn.com/abstract=3680955. Accessed 14 September 2020.

6. Gupta V, Bhoyar RC, Jain A, Srivastava S, Upadhayay R, Imran M et al. Asymptomatic reinfection in two healthcare workers from India with genetically distinct SARS-CoV-2. Clinical Infectious Diseases , ciaa1451, https://doi.org/10.1093/cid/ciaa1451

7. Lancman, G., Mascarenhas, J. \& Bar-Natan, M. Severe COVID-19 virus reactivation following treatment for B cell acute lymphoblastic leukemia. J Hematol Oncol 13, 131 (2020). 
https://doi.org/10.1186/s13045-020-00968-1

8. Yadav S, Wadhwa T, Thakkar D, et al. Covid19 Reinfection in Two Children with Cancer. Authorea. September 11, 2020. DOI: 10.22541/au.159986505.57940176

9. Radhakrishnan, V. and Gangopadhyay, D. (2020), Repeat-positive SARS-CoV-2 in a child with cancer. Pediatr Blood Cancer. doi:10.1002/pbc.28744

10. Bhoyar RC, Jain A, Sehgal P, Divakar MK, Sharma D, Imran M et al. High throughput detection and genetic epidemiology of SARS-CoV-2 using COVIDSeq next generation sequencing. bioRxiv 2020.08.10.242677; doi: https://doi.org/10.1101/2020.08.10.242677

11. Poojary M, Shantaraman A, Jolly B, Scaria V. Computational Protocol for Assembly and Analysis of SARS-nCoV-2 Genomes Research Reports 4: e1-e14 doi:10.9777/rr.2020.10001

\section{Legend}

Fig. 1. Variants in the genome isolates from the two episodes E1 and E2 with their respective read count proportions. 


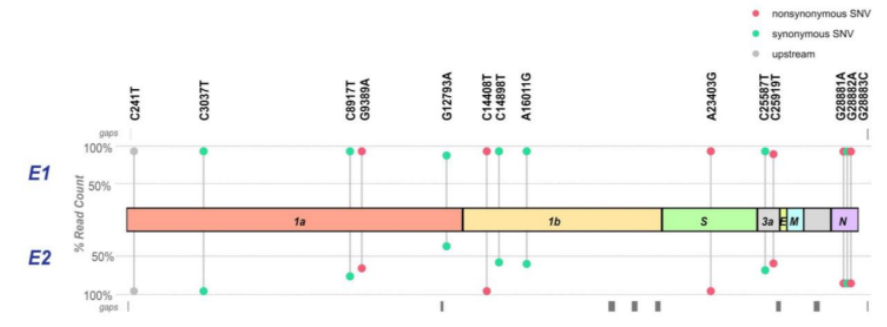

